\title{
Management of pediatric penile keloid
}

\author{
Michael Yong, BSc; ${ }^{*}$ Kourosh Afshar, MD, FRCSC; ${ }^{\dagger}$ Andrew MacNeily, MD, FRCSC; ${ }^{\dagger}$ Jugpal S. Arneja, MD, \\ $M B A, F A A P, F A C S, F R C S C^{\varsigma}$
}

"Faculty of Medicine, University of British Columbia, Vancouver, BC; 'Division of Pediatric Urology, British Columbia Children's Hospital and University of British Columbia, Vancouver, BC; §Division of Plastic Surgery, British Columbia Children's Hospital and University of British Columbia, Vancouver, BC

Cite as: Can Urol Assoc J 2013;7(9-10):e618-20. http://dx.doi.org/10.5489/cuaj.408

Published online September 10, 2013.

\section{Abstract}

Keloids are abnormal scar tissue growths that extend beyond the original area of injury, occasionally occurring post-surgery. Risk factors for keloids include skin trauma, infection, prolonged wound healing and individuals of certain ethnicities. Keloid formation on the penis, however, is a rare occurrence even among circumcised males, and can produce both aesthetic and functional complications. We document the tenth patient in the literature, to our knowledge, to present with a keloid of the penis.

\section{Introduction}

Keloids are abnormal scar tissue growths that extend beyond the original area of injury, occasionally occurring post-surgery. Risk factors for keloids include skin trauma, infection and prolonged wound healing. Keloid formation on the penis, however, is a rare occurrence even among circumcised males, and can produce both aesthetic and functional complications. We document the tenth patient in the literature, to our knowledge, to present with a keloid of the penis.

\section{Case report}

A 19-month-old male of African descent was referred to us with a keloid of the shaft of the penis. The patient had undergone a circumcision in the neonatal period and had since developed a thickened tumour. The patient's mother describes wound separation post-circumcision, with several weeks before ultimate healing. The patient was asymptomatic from a urologic point of view, but the lesion was not aesthetically acceptable to the family. The patient, of African descent, had no previous or family history of keloid formation. Given the extreme size of the tumour (Fig. 1, Fig. 2) and to prevent future complications, we decided to excise the keloid and reconstruct this either with a skin graft or via advancement of the local tissues.

An incision was established with a 1-mm margin around the tumour through skin to the level of the dartos fascia. The keloid, measuring $4.6 \mathrm{~cm} \times 1.8 \mathrm{~cm}$, was dissected off of the dartos fascia circumferentially, and then excised in its entirety and sent to pathology for further histopathological review (Fig. 3, Fig. 4). The dartos fascia was not breached nor was the urethra entered.

For reconstruction, a small lip of normal tissue was left at the ventral base of the corona resembling a circumcisionstyle incision. Proximally, for about $2 \mathrm{~cm}$ circumferentially, the level of dartos fascial layer was undermined for tensionfree advancement of the penile shaft skin. The proximal tissues were able to be advanced to the corona without any evidence of tension. As such, reconstruction and closure of the skin followed. Monocryl 5-0 interrupted and running intracuticular sutures were used to close the wound (Fig. 5). There were no intraoperative complications and dressings consisted of bacitracin ointment only. The patient was discharged on postoperative day 1 . The patient was recurrence-free 3 years postoperatively (Fig. 6). The patient had no postoperative complications related to urologic function. The histopathology was suggestive of a keloid and no other abnormalities were found upon analysis.

\section{Discussion}

Keloids are benign, hyperproliferative scar tissue growths, characterized by excessive deposition of collagen and other extracellular matrix components. ${ }^{1,2}$ Keloid differs from hypertrophic scar in that it extends beyond the original wound margins and does not resolve spontaneously. Often arising secondary to operative procedures, risk factors for keloid 


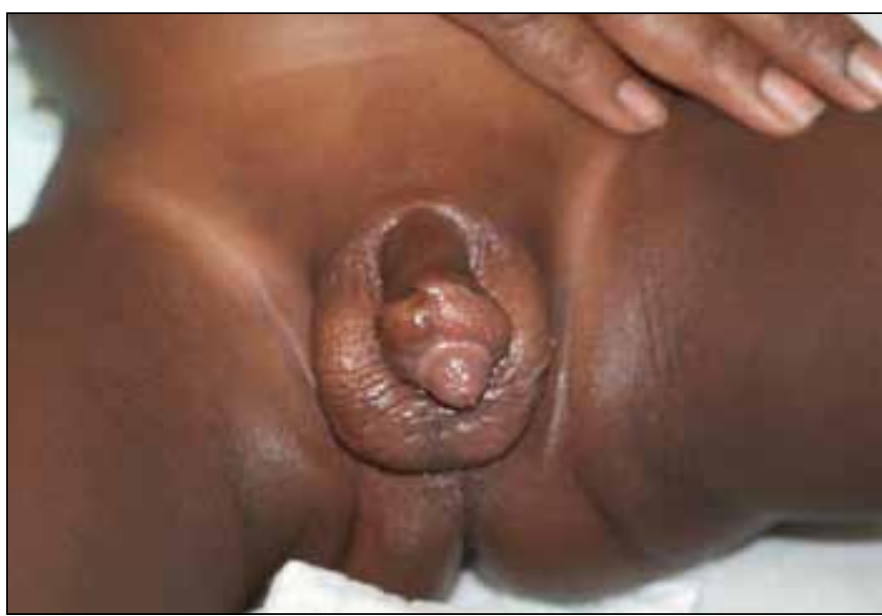

Fig. 1. A 19-month male with a post-circumcision keloid of the penile shaft.

formation include skin trauma, prolonged wound healing, infection, wounds in certain anatomical regions and foreignbody reactions, especially in genetically susceptible individuals. ${ }^{2,3}$ Although the exact pathogenetic mechanisms are still unknown, extracellular matrix abnormalities, aberrant collagen turnover, mechanical tension and genetic immune dysfunction have all been proposed as pathogenetic hypotheses. ${ }^{1}$ In addition, fibroblast cells derived from keloid tissue display increased proliferation and density, among many other characteristics. Over-expression of growth factors, such as TGF- $\beta$ in fibroblasts, has been proposed as a major contributor to the pathogenesis of keloid formation. ${ }^{1,3}$ The most likely cause of this patient's keloid was post-circumcision dehiscence resulting in prolonged wound healing in a genetically predisposed individual.

Keloids can vary in size from millimetres to centimetres in diameter, and are most common in areas, such as the posterior shoulder, presternal area, earlobes and posterior

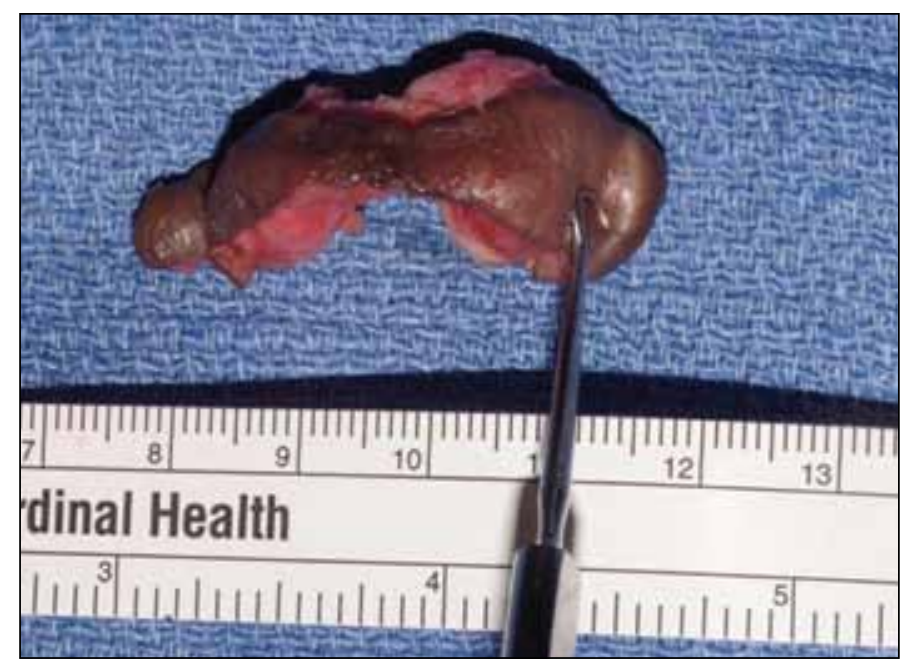

Fig. 3. Penile shaft keloid lesion, excised specimen.

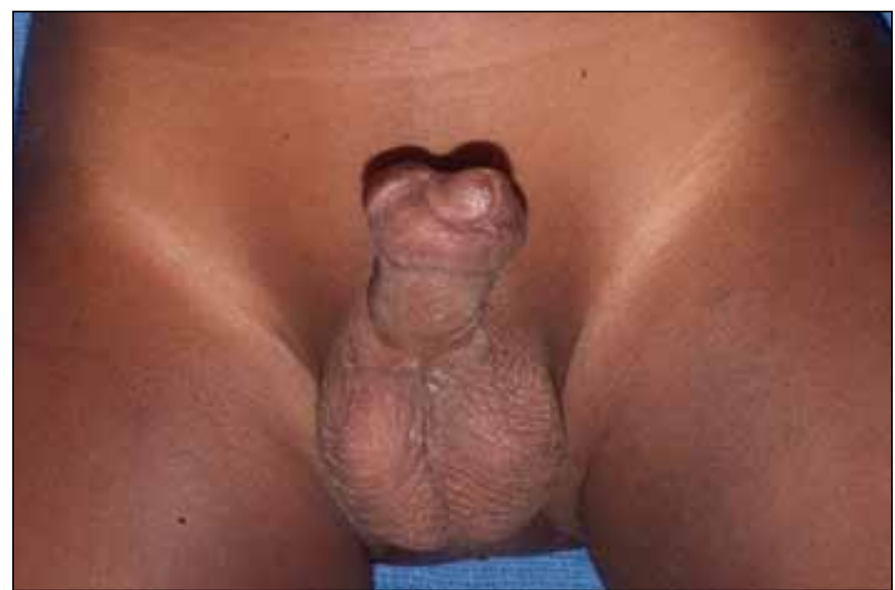

Fig. 2. Penile shaft keloid lesion, pre-incision.

neck. Keloids have the potential to be complicated by pruritus, tenderness, burning, secondary infection, ulceration and restriction of motion. ${ }^{4,5}$ The penis is frequently subjected to surgical trauma, such as circumcision, but keloid formation is not a common complication or occurrence. Although keloid formation has been documented to be most frequent in patients between the ages of 15 and 45 years old, ${ }^{6}$ our patient presented with a penile shaft keloid at the age of 19 months. In the pediatric urology patient population, keloids are rare entities; in a series studying hypospadias repair outcomes, Nitkunan and colleagues reported only 1 case of keloid formation in a series of 104 patients who had two stage repairs. ${ }^{7}$

Several modes of treatment exist for keloids; the most common therapy is surgical excision coupled with intralesional steroid injection and radiation therapy. ${ }^{1}$ Due to the close proximity of germ cells, though, radiation has not been a desirable therapy for penile keloids. Local recurrence rates of keloids removed by surgical excision alone can be significant, but surgery combined with injection(s) of corticosteroids can reduce the local recurrence rates to

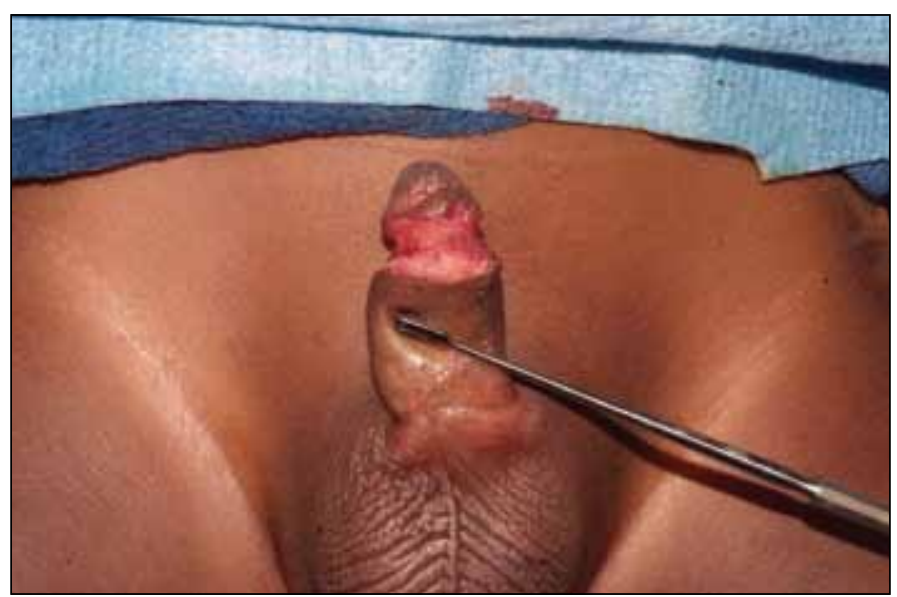

Fig. 4. Penile shaft, post-excision. 


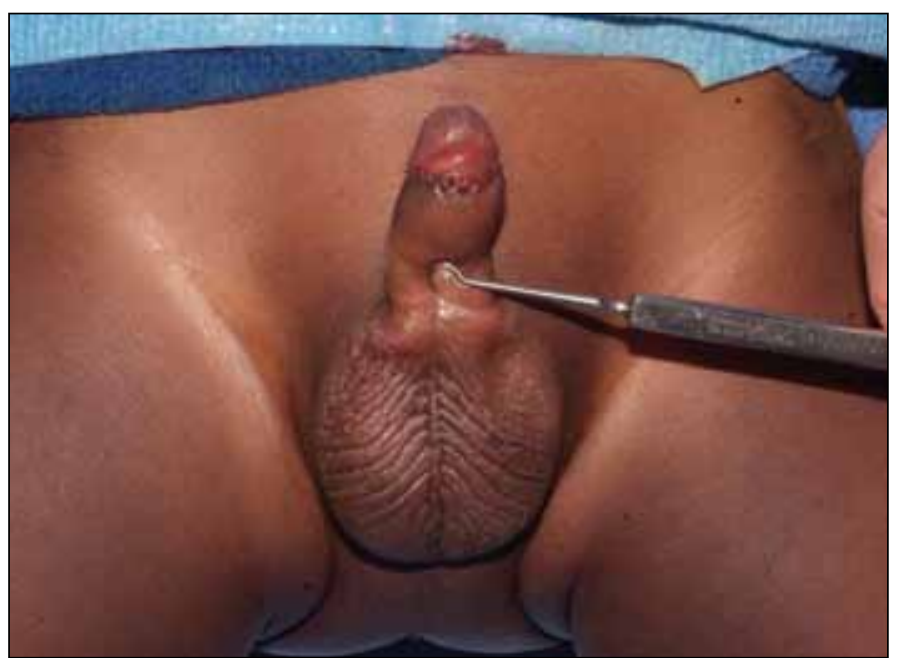

Fig. 5. Penile shaft, post advancement of shaft skin, following closure.

below $50 \% .^{8}$ However, steroid treatment carries the risk of adverse effects, including subcutaneous atrophy, telangiectasis, pigment changes and systemic side effects. ${ }^{5}$ Complete surgical excision with advancement of local tissues was the treatment administered to our patient. No intralesional steroids were administered considering the young age of our patient and potential complications arising from steroid administration. Our patient remained recurrence-free 3 years postoperatively. Untreated, this could have produced functional complications in the future, including aesthetic discomfort, abnormal erection, sexual dysfunction and voiding difficulties.

\section{Conclusion}

We report the successful management, 3 years postoperatively, of a penile shaft keloid excision with advancement reconstruction. Because our treatment did not involve administration of intralesional steroids, long-term complications were potentially avoided. Keloid is an uncommon sequelae of circumcision and should be treated aggressively to avoid potential future functional complications.

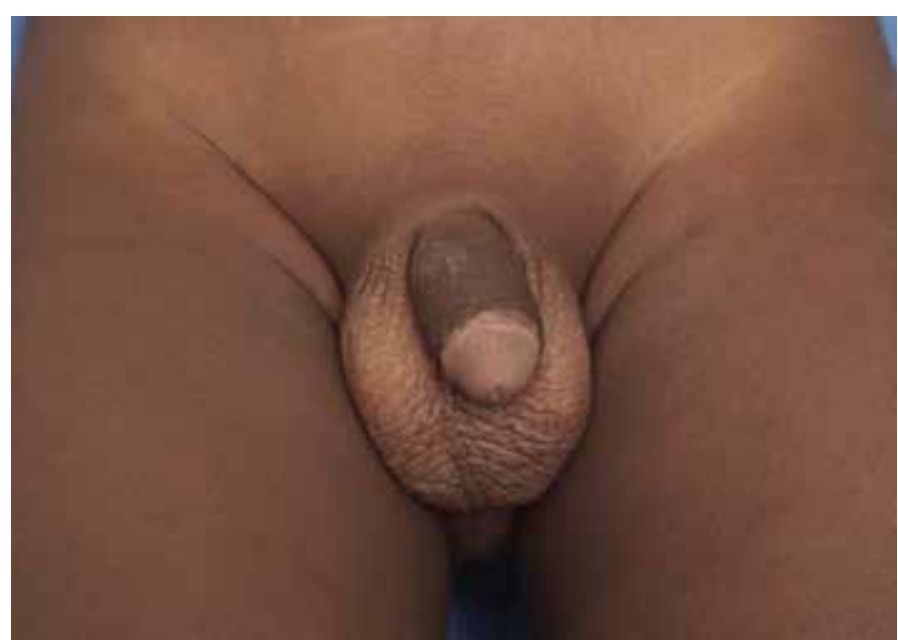

Fig. 6. Penile shaft, 3 years postoperatively, recurrence-free.

\section{References}

1. Marneros AG, Krieg T. Keloids - clinical diagnosis, pathogenesis, and treatment options. J German Society Dermatol 2004;2:905-13.

2. Kelly AP. Medical and Surgical therapies for keloids. Dermatol Ther 2004;17:212-8. http://dx.doi. org/10.1111/j.1396-0296.2004.04022.x

3. Al-Attar A, Mess S, Thomassen JM, ET AL. Keloid pathogenesis and treatment. Plast Reconstr Surg 2006;117:286-300. http://dx.doi.org/10.1097/01.prs.0000195073.73580.46

4. Rockwell WB, Cohen IK, Ehrlich HP. Keloids and hypertrophic scars: a comprehensive review. Plast Reconstr Surg 1989;84:827-37. http://dx.doi.org/10.1097/00006534-198911000-00021

5. Alster TS, Tanzi EL. Hypertrophic Scar and Keloids: Etiology and Management. Amer J Clin Dermatol 2003;4:235-43. http://dx.doi.org/10.2165/00128071-200304040-00003

6. Gurunluoglu $R$, Bayramicli M, Numanoglu A. Keloid of the penis after circumcision. Br I Plast Surg 1996;49:425-6. http://dx.doi.org/10.1016/S0007-1226(96)90018-1

7. Nitkunan T, Johal N, $\mathrm{O}^{\prime}$ Malley K, et al. Secondary hypospadias repair in two stages. J Pediatr Urol 2006;2:559-63. http://dx.doi.org/10.1016/i.jpurol.2006.01.009

8. Erdemir $F$, Gokce 0 , Sanli 0 , et al. A rare complication after circumcision:keloid of the penis. Int Urol Nephrol 2006;38:609-11. http://dx.doi.org/10.1007/s11255-006-0021-6

Correspondence: Dr. Jugpal S. Arneja, Associate Professor, Surgery (Clinical)- University of British Columbia, Associate Chief, Surgery, British Columbia Children's Hospital Division of Plastic Surgery, A237 Shaughnessy Bldg, 4480 Oak St., Vancouver, BC V6H 3V4; fax: 604-875-2749; jugpal.arneja@ubc.ca

Competing interests: None declared.

This paper has been peer-reviewed. 